\title{
A study to further develop and refine carpal tunnel syndrome (CTS) nerve conduction grading tool
}

\author{
Salim Hirani(0)
}

\begin{abstract}
Background: The severity of carpal tunnel syndrome (CTS) may be categorised in a number of ways utilising one of a range of presently available grading tools. The grading systems proposed by Bland and Padua are the most commonly used, however, both have limitations, which are discussed in detail in this paper.

The aim of this research is to establish, using the best available evidence, a clinically appropriate revision of the current CTS nerve conduction grading tool, and to compare with existing grading tools used in UK Neurophysiology clinics. The revised scale is designed from a clinical physiologist perspective and based on the numerical values of nerve conduction findings.

The proposed revised grading system is based on more nuanced, descriptive categories, ranging from Normal to Early, Mild Sensory, Mild Sensory Motor, Moderate Sensory, Moderate Sensory Motor, Severe Sensory Motor, Extremely Severe Sensory Motor, and Complete absence.

Method: A total of 1123 patients (2246 hands) were included in this study, with the aim of evaluating the revised grading system. Data was collected based on the extensive and detailed grading systems previously described by Bland and Padua. All data was recorded numerically to ensure methodological reliability.

Result: Of the 2246 patients' hands tested, the nerve conduction was graded as normal in 968 hands; nerve conduction showed early changes in 271 hands; mild sensory changes in 215 hands, mild changes in both motor and sensory response in 51 hands; moderate sensory changes in 134 hands; moderate sensory and motor changes in 356 hands; severe changes in motor and sensory responses in 204 hands; extremely severe sensory and motor changes in 33 hands and complete absence of response in 14 hands.

Conclusion: The revised grading tool could offer a more numerical grading to the Clinical Physiologist and could help the surgeon to ascertain the level of severity in order to decide on either a conservative or surgical approach to treatment if they decide to use the proposed grading which could support them to defend their decision in cases of litigation.
\end{abstract}

Keywords: Grading tools for carpal tunnel syndrome, CTS Gradings, Neurophysiological CTS grading

Correspondence: salimwali_mohd@hotmail.com

Neurophysiology Department, Ysbyty Gwynedd Hospital, Bangor, North

Wales LL57 2PW, UK

(c) The Author(s). 2019 Open Access This article is distributed under the terms of the Creative Commons Attribution 4.0 International License (http://creativecommons.org/licenses/by/4.0/), which permits unrestricted use, distribution, and reproduction in any medium, provided you give appropriate credit to the original author(s) and the source, provide a link to the Creative Commons license, and indicate if changes were made. The Creative Commons Public Domain Dedication waiver (http://creativecommons.org/publicdomain/zero/1.0/) applies to the data made available in this article, unless otherwise stated. 


\section{Background}

The pathology of Carpal Tunnel Syndrome (CTS) is described as "A Neuropathy caused by entrapment of the median nerve at the level of the carpal tunnel" $[1,2]$. Nerve Conduction Studies (NCS) are one of the basic tools used to support clinical diagnosis. NCS are objective tests that assess the physiological status of the median nerve across the carpal tunnel [3].

\section{Reason for grading carpal tunnel syndrome}

The Grading tool is used for the diagnostic assessment of CTS in conjunction with the patient's clinical history and symptoms in order to diagnose the degree of severity of CTS [2]. The revised grading tool using a physiological basis offers a more precise numerical grading, which is both objective and repeatable. This could not only help the Clinical Physiologist to grade there result according to the propose grading scale but probably it also support the surgeon to ascertain the level of severity and could help to decide on either a conservative or surgical approach to treatment.

There are several primary grading tests mentioned in the different literature, associated with Phalen's, Tinel's and Durkan's signs which are subjective and are based on patient clinical response. Other tests like Ultrasound, NCS and EMG needle examination are objective tests that have been used for CTS grading which are reliable, evidence-based and objective, not dependent on patient clinical response [1].

However, to ascertain the severity level of CTS, specific neurophysiological grading is required [4]. There are several grading scales for investigations specifically related to CTS; [Campbell [5], Padua [4], Bland [6], Giannini [3], Carvalho [7], Ajeena [1], Jeong [8] and Jerosh-Herold [9]]. Most of the studies show grading in subjectivity. Some lack a neurophysiological focus in objectivity [7] during the collection of the data. Some researches only use either Sensory Nerve Conduction Study (NCS) or Motor NCS to differentiate the severity of CTS grading. Not all researchers have used sensitive techniques to diagnose early CTS or in severe cases, Lumbrical responses to differentiate its severity from complete absence, which therefore cannot be diagnosed as CTS with complete certainty.

It appears that whilst there is an accepted dominance of both the Bland [6] and Padua [4] grading systems, there are also clear limitations which are discussed comprehensively in this paper.

In the UK, the Bland [6] grading is largely followed due to its depth of detail. In 2014 the Association of Neurophysiological Science (ANS), in collaboration with the British Society for Clinical Neurophysiology (BSCN) published guidelines outlining the accepted grading of CTS in the United Kingdom, which follows the Bland
[6] grading system. The reason given was that it focuses on the clinical physiologist specialism, as well as its element of flexibility.

The aim of this research was to establish evidencebased revision of the current CTS nerve conduction Grading Tool used in the UK and to evaluate its effectiveness - in terms of acceptability and usability for Clinical Physiologist as well as a tool for intervention prediction for Surgeon. This could support the Surgeon to ascertain the level of severity and decide on a conservative or surgical approach to treatment. Although surgeons must take their own decision for the treatment of CTS, if they want to consider the treatment on the basis of the proposed Nerve conduction study grading, this will probably allow to defend their decisions in the Magistrate Court. A numerical value is given to each of the grade bandings to enable objective reporting and comparision [4].

No clinical assessment was conducted during the Neurophysiological test so as to secure the biasness from patient's condition.

\section{Method}

Ethical approval for the research project was obtained from the Heath Research Authority National Research Ethics Service London - Queen Square Research Ethics Committee (Reference 17/LO/0750).

Neurophysiological data was collected based on the extensive and complete description of previous study designs by Padua [4] and Bland [6] and which is understood to be followed by most of the clinical laboratories in the United Kingdom. In addition, Second LumbricalInterosseous Latency was also recorded to distinguish between 'very severe' and 'complete absent' response grading of CTS [10].

The Association of Neurophysiological Scientists (ANS) (2014) guidelines and the minimum standards for the practice of Clinical Neurophysiology in the United Kingdom were followed. Few new grading was introduced during collection of the data to cover full range of grading.

The test was performed by a qualified Clinical Physiologist (Neurophysiology) using Keypoint 9033A07 (Skovlunde, Denmark) machine, on the bases of departmental protocol (Peripheral protocol1, 2015). A quantitative method was used for collecting data [11], to ensure accuracy and to avoid bias. The sample size of patients in the study was use for all those tested for NCS over a period of one calendar year (2017), across the population of North Wales. The data was collected from patients with an age range above 18 years, who were referred to the Neurophysiology department from the Orthopaedics and Neurology departments within the local Health Board, as well as General Practices (GPs) in 
North Wales. No individual patient was recruited in this research. The inclusion criteria were considered only on the basis of the referral diagnosis. No clinical assessment was conducted prior to the study in the department. Referral of CTS was considered based on paraesthesia, pain, swelling in median distribution area or digits I-V, worsened by sleep. The test was carried out by testing both hands (symptomatic and asymptomatic) to fulfil department protocol.

Data was analysed on certain widely accepted assumptions of sensory amplitude and CV and distal motor latency (DML), amplitude and CV $[2,6]$. To introduce the terms "mild", "moderate" and "severe", a numerical value was used which could be accepted widely, and which can be used to compare with others studies [4].

The procedure started by carrying out the sensory testing, by placing the stimulating ring electrodes on digit III (which is more sensitive then digit II [6]) and the recording electrode on the surface of the median nerve on the wrist. The orthodromic technique was used for the sensory and motor NCS test, through the median and ulnar nerves. A maximal current was applied to record the full response of the nerve, at the digits II-IV for median sensory and digit $\mathrm{V}$ for ulnar sensory recording. A maximal current was applied to stimulate median nerve pathways at the wrist and at the elbow for motor recording from abductor pollicis brevis (APB) [11] and ulnar nerve pathways from First dorsal interosseous (FDI). Digit II was stimulated only when either the response from digit III was less than $3 \mu \mathrm{V}$ or absent; digit IV was stimulated only when the response from digit III showed conduction velocity between 45 and $50 \mathrm{~m} / \mathrm{sec}$. Amplitude was recorded from peak to peak for sensory responses, and base to peak for motor responses. If responses were not recordable from median sensory digit II, III and motor from APB muscles, then motor responses were elicited by placing recording electrodes on 2nd lumbricals by stimulating median and ulnar nerves at the wrist [7, 10-12].

All patient data was collected by fulfilling the criteria mentioned in above paragraph depending on the severity. The reason for using the new criteria is to describe the full range of severity which was not fully covered by other research mentioned earlier in this paper. Criteria was mentioned in above paragraph are intended to be more reliable in terms of grading for Clinical Physiologist and probably will allow support to the Surgeon in terms of patient treatment decisions.

The grades are:

Normal (Grade 0): where sensory conduction velocity (SCV) is above $50 \mathrm{~m} / \mathrm{s}$ and amplitude $\geq 5 \mu \mathrm{V}$ with DML $\leq 4.2 \mathrm{~ms}$, amplitude $\geq 5 \mathrm{mV}$ and motor conduction velocity $(\mathrm{MCV}) \geq 50 \mathrm{~m} / \mathrm{s}$.
Early (Grade 1): where SCV is between 45 and $50 \mathrm{~m} / \mathrm{s}$ from digit III and double peak latency in digit IV is > $0.5 \mathrm{~ms}$ with DML $\leq 4.2 \mathrm{~ms}$ and normal sensory and motor amplitude $>5$ (sensory in $\mu \mathrm{V}$ and motor in $\mathrm{mV}$ ).

Mild Sensory (Grade 2): where SCV is between 40 and $44.9 \mathrm{~m} / \mathrm{s}$ from digits III with normal sensory amplitude and motor values mentioned in Grade 0.

Mild Sensory-Motor (Grade 3): where SCV is between 40 and $44.9 \mathrm{~m} / \mathrm{s}$ from digits III with normal sensory amplitude mentioned in Grade 0, DML $\geq 4.2 \mathrm{~ms}$ with normal motor amplitude and CV.

Moderate Sensory (Grade 4): where SCV is less than $40 \mathrm{~m} / \mathrm{s}$ from digits III with normal sensory amplitude and normal motor values mentioned in Grade 0.

Moderate Sensory-Motor (Grade 5): where SCV is less than $40 \mathrm{~m} / \mathrm{s}$ from digits III with normal sensory amplitude, DML $\geq 4.2 \mathrm{~ms}$ with normal motor amplitude and $\mathrm{CV}$.

Severe Sensory-Motor (Grade 6): where sensory potentials from digits III and digit II are absent or $<3 \mu \mathrm{V}$ in both digits III and II with $\mathrm{SCV}<30 \mathrm{~m} / \mathrm{s}, \mathrm{DML} \geq 4.2 \mathrm{~ms}$, $\mathrm{MCV}$ is either slow or normal.

Extremely Severe Sensory-Motor (Grade 7): where sensory and motor potentials are absent and response recordable only from 2nd lumbricals, where median lumbricals are prolonged compared and low amplitude to ulnar lumbricals.

Complete (Grade 8): where both sensory and motor potentials are absent and responses are not recordable from median 2nd lumbricals but recordable from ulnar 2nd lumbricals. (Please refer to a Comparison of the Bland [6] grading with the proposed revised grading is given at the end of this study for more understanding).

\section{Results}

The data was collected for a period of 1 year (2017). Initially a total of 1132 patients were included in this study. During data collection, two referrals were not included, because the patients declined to participate in all study procedures; and seven participants' data sets were excluded from the analysis because the departmental protocol was breached. Therefore 1123 patients (2246 hands) were included in the final data collection.

Of the 1123 patients, 687 were female and 436 were male. The age range was 19 to 98 years, median age 56 years. The numbers of hands in each grade of severity are shown in Fig. 1 and Table 1.

\section{Discussion}

The Bland [6] grading system, which was collected for Canterbury region, enables the neurophysiologist to differentiate between the levels of severity for the Clinical Physiologist point of view [4]. However, due to the limited numerical grading, it is felt that the Bland [6] 


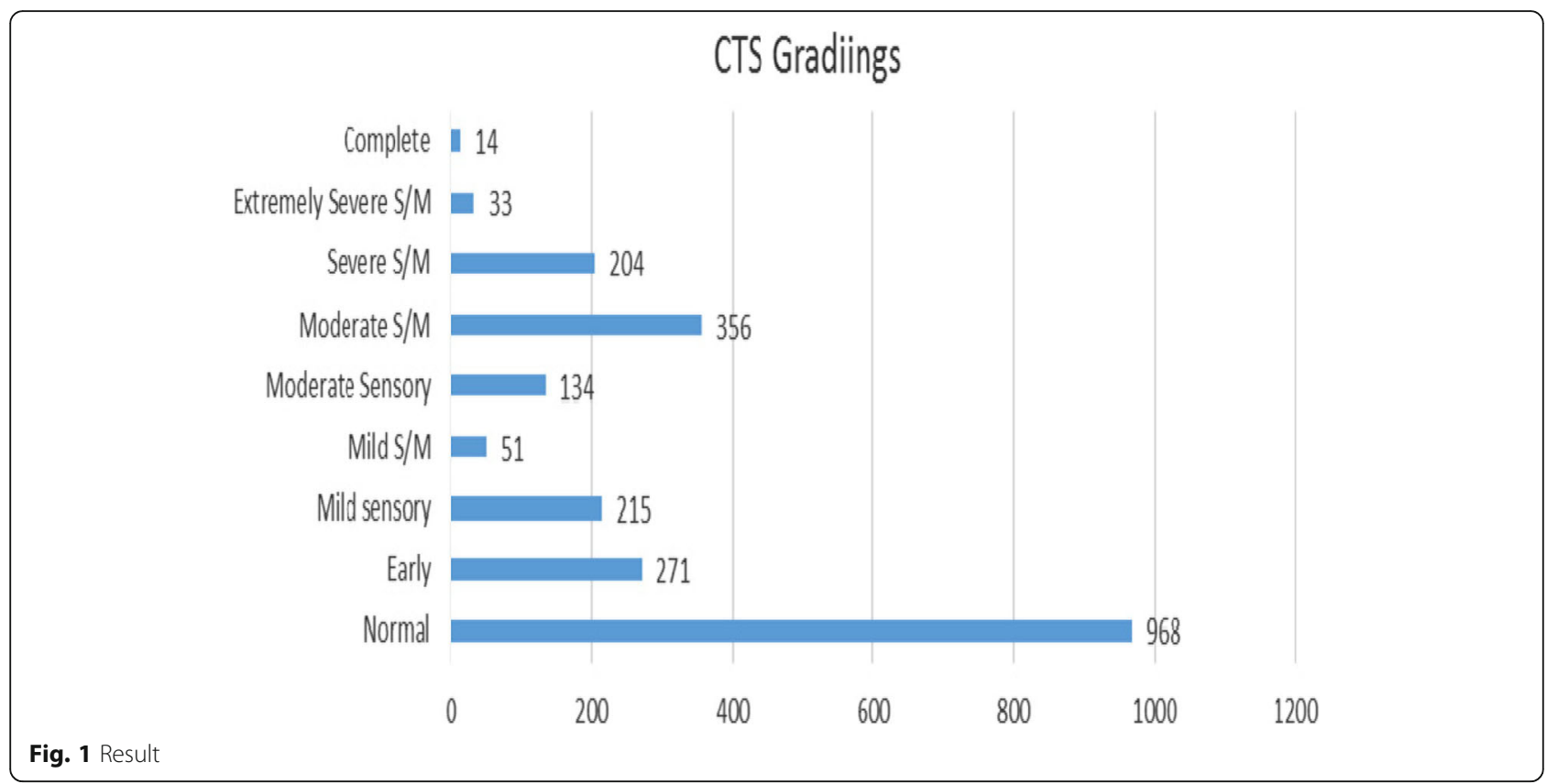

grading does not enable the level of severity to be objectively and fully ascertained as possible to cover all level of grading of CTS.

Bland [6] recorded that prolongation of the motor terminal latency to APB is not significant in mild cases which the author partially agrees with, as it appears insignificant in the graph (Please refer to the graph) compared to mild sensory CTS. It was noted that neither Bland [6] or Padua [4] separate between mild sensory and mild sensory-motor in their Grading. This clearly indicates that there is a need for some revision and a separate grading in mild CTS. However, when compared to moderate sensory- motor CTS with moderate sensory CTS, moderate sensory-motor CTS has significantly higher patient numbers than the moderate sensory. This indicates that there is room for revision and a separate grading in moderate CTS. (Please refer to percentage table).

Table 1 Result

\begin{tabular}{ll}
\hline Normal & 968 \\
Early & 271 \\
Mild sensory & 215 \\
Mild S/M & 51 \\
Moderate Sensory & 134 \\
Moderate S/M & 356 \\
Severe S/M & 204 \\
Extremely Severe S/M & 33 \\
Complete & 14 \\
Total Hands & 2246 \\
\hline
\end{tabular}

In theory, the higher the grade, the worse nerve dysfunction $^{4}$. The analysis of the data, however, in this study appears to show mixed levels of severity. A majority of CTS studies in this investigation appear normal, due to the fact that non-symptomatic hands were also recorded to fulfil department protocol. Although normal (Grade 0) has much higher numbers compared to other grades. This does not detract from the fact that levels of severity for CTS were found. In this study, comparison of the grading scales shows that there are big differences in the mild sensory-motor groups between Bland's [6] and this proposed grading. $11 \%$ in Bland's [6] mild sensory-motor category with $10 \%$ mild sensory and only $2 \%$ mild mixed sensory motor in propose grading, which might suggest that the separation is marked, although the separation has value in demonstrating the effects in the motor fascicles and this may have an impact in choosing treatment option. However, at the moderate degree of severity, there is a notable difference with $16 \%$ in Bland's [6] moderate category and 6\% moderate sensory and $16 \%$ moderate mixed sensory motor, suggestive that these are 2 groups worth separating.

The Table 2 summarises and compares the variance in Bland [6] and Padua [4] Gradings with the revised grading system.

Padua [4] relates the outcome of grading with surgical decompression compared to Bland's [6] grading which is based purely on neurophysiological concepts. Bland's [6] grading scale was based on a very large population. However, in a 'severe' grading, the values were not clear. Bland [6] has given the amplitude for the motor response, but has not taken into account the DML and CV, and for 
Table 2 Percentage comparison with grading of Padua, Bland and Hirani

\begin{tabular}{|c|c|c|c|}
\hline \multicolumn{2}{|l|}{ Padua (\%) } & \multirow{2}{*}{$\begin{array}{l}\text { Bland (\%) } \\
\text { Normal = } 3269(38)\end{array}$} & \multirow{2}{*}{$\begin{array}{l}\text { Hirani (\%) } \\
\text { Normal =968 (43) }\end{array}$} \\
\hline & Normal = 18 (3) & & \\
\hline & Minimal = $123(21)$ & Very mild = 684 (8) & Early $=271(12)$ \\
\hline & \multirow[t]{2}{*}{ Mild = $145(24)$} & \multirow[t]{2}{*}{ Mild sensory-motor $=944(11)$} & Mild sensory = $215(10)$ \\
\hline & & & Mild S/M = 51 (2) \\
\hline & \multirow[t]{2}{*}{ Moderate = 217 (36) } & \multirow[t]{2}{*}{ Moderately Severe =1359 (16) } & Moderate sensory = 134 (6) \\
\hline & & & Moderate S/M = 356 (16) \\
\hline & Severe $=81(14)$ & Severe $=568(7)$ & Severe $S / M=204$ (9) \\
\hline & \multirow[t]{2}{*}{ Extremely severe $=16(3)$} & Very severe = 930 (11) & Extremely Severe $S / M=33$ (1) \\
\hline & & Extremely Severe = 387 (5) & Complete $=14(1)$ \\
\hline Total Hands & 600 & 8501 & 2246 \\
\hline
\end{tabular}

'extremely severe' grading the values was not clear either. At 'extremely severe' grading, although the amplitude in the motor CMAP has been taken into account, no account has been taken of distal latency and CV [6].

Comparing, the Bland [6] grading system of CTS with the grading suggested in this paper, it seems that Bland [6] Grade 0 and 1 are comparable with the propose grading. The author would suggest that the Bland [6] grading for Grade 2 needs to become more elaborate by dividing them into two separate groups, i.e. mild sensory and mild sensory-motor, as more patient's data shows just mild sensory changes compared with mild sensory and motor together. In the revised grading the sensory involvement is graded as Grade 2 and the sensory and motor where both functions are involved is graded as Grade 3.

Bland [6] covers a moderate degree of severity in grade 3 , which again warrants further elaboration to make the gradings more objective and more descriptive. In propose revised system grade 4 covers sensory involvement and grade 5 covers both sensory and motor involvement together.

Bland's [6] Grade 4 which is a severe CTS compares favourably with Grade 6 of the revised grading system.

Bland [6] only describes a prolonged DML in his Grade 5 as 'very severe' CTS which the author believes do not fulfil all the criteria to separate from his Grade 6 . Bland [6] appears not to have taken any account changes in sensory potentials or motor conduction velocity values. The revised grading system has graded 'Very Severe' CTS where both sensory and motor responses are absent, and responses were only recordable from the 2nd lumbrical with prolonged median distal latency compared to ulnar lumbricals as Grade 7.

The Grade 6 in Bland [6] again has the potential to create confusion as it refers to a low amplitude motor

Table 3 Grading comparison of Bland with propose grading

\begin{tabular}{|c|c|c|}
\hline Grading & Bland [6] & Modified grading by Hirani \\
\hline Grade1 & $\begin{array}{l}\text { Inching, palm/wrist median/ulnar comparison, ring } \\
\text { finger double peak }\end{array}$ & $\begin{array}{l}\text { Early: SCV }=45-50 \mathrm{~m} / \mathrm{s} \text { interpeak potentials in digit IV }>0.5 \mathrm{~ms}, \mathrm{DML}<4.2 \mathrm{~ms} \text {. } \\
\text { Amplitude of sensory } \geq 5 \mu \mathrm{V} \text { and motor potentials } \geq 5 \mathrm{mV}\end{array}$ \\
\hline Grade2 & $\begin{array}{l}\text { Mild: sensory conduction velocity }(\mathrm{SCV})<40 \mathrm{~m} / \mathrm{s} \\
\text { distal motor latency }(\mathrm{DML})<4.5 \mathrm{~ms}\end{array}$ & $\begin{array}{l}\text { Mild sensory: } \mathrm{SCV}=40-44 \mathrm{~m} / \mathrm{s} \text { with normal sensory amplitude (NSA), DML, motor } \\
\text { nerve action potentials (MNAP) and sensory conduction velocity (SCV) \& motor } \\
\text { conduction velocity (MCV) }\end{array}$ \\
\hline Grade3 & $\begin{array}{l}\text { Moderately severe: DML }>4.5 \mathrm{~ms} \text { and }<6.5 \mathrm{~ms} \text { with } \\
\text { sensory nerve action potentials (SNAP) preserved }\end{array}$ & $\begin{array}{l}\text { Mild sensory motor: } \\
\text { SCV }=40-44 \mathrm{~m} / \mathrm{s} \text { with NSA, DML }>4.2 \mathrm{~ms} \text { with normal motor amplitude (NMA) } \\
\text { and normal SCV and MCV }\end{array}$ \\
\hline Grade4 & Severe: $\mathrm{DML}>4.5 \mathrm{~ms}$ and $<6.5 \mathrm{~ms}$ with absent SNAP & Moderate sensory: SCV $<40 \mathrm{~m} / \mathrm{s}$ with NSA, normal DML, NMA, SCV and MCV \\
\hline Grade5 & Very severe: DML > $6.5 \mathrm{~ms}$. & $\begin{array}{l}\text { Moderate sensory motor: SCV }<40 \mathrm{~m} / \mathrm{s} \text { with NSA, DML }>4.2 \mathrm{~ms} \text {, MNA and SCV } \\
\text { and MCV }\end{array}$ \\
\hline Grade6 & $\begin{array}{l}\text { Extremely Severe: motor nerve action potentials } \\
(\mathrm{MNAP})<0.2 \mathrm{mV} \text {, }\end{array}$ & $\begin{array}{l}\text { Severe Sensory motor: Absent or }<3 \mu \mathrm{V} \text { SNAP with } \mathrm{SCV}<30 \mathrm{~m} / \mathrm{s} \text { with } \mathrm{DML}>4.2 \\
\text { ms with either slow or normal MCV and or NMA }\end{array}$ \\
\hline Grade7 & & $\begin{array}{l}\text { Extremely severe: SNAP and MNAP = absent, but recordable from both median } \\
\text { and ulnar } 2 \text { nd lumbricals with prolonged median } 2 \text { nd lumbricals response as } \\
\text { compare to ulnar lumbricals }\end{array}$ \\
\hline Grade8 & & $\begin{array}{l}\text { Complete: } \\
\text { SNAP and MNAP = absent and absent from median 2nd lumbricals and present } \\
\text { from ulnar 2nd lumbricals }\end{array}$ \\
\hline
\end{tabular}


potential. It appears not to have taken into account the DML in Grade 6. CV was also not included in the Grade 6 grouping in the Bland [6] grading. The author has considered the latency and amplitude with $\mathrm{CV}$ and graded as complete median nerve dysfunctions where both median motor and sensory as well as median 2nd lumbrical responses are absent, and the only response appears in the ulnar 2nd lumbrical. This grade appears as grade 8 in the revised grading system.

Table 3 summarises and compares the Bland [6] Gradings with the revised grading system.

\section{Conclusion}

The grading system devised by Bland [6] and used to grade the levels of severity of CTS over the last 17 years within the UK has certain limitations, and the author believes system needs modification in order to accommodate current practice. The revised grading system for CTS is based on a review of a current and past literature.

Bland [6] and Padua [4] both limited the DML and $\mathrm{CV}$ in motor study and amplitude potentials and $\mathrm{CV}$ for sensory study. Author follow the same rule and precedes the study with given cut off values to grade them accordingly. Most of the Clinical laboratories in UK use the above criteria of cut off values for sensory and motor study to create their own normative values. Presently, there is no standard of CTS grading followed throughout the UK due to their limitations, the propose grading scale, preferably was felt to be an acceptable and useable tool for Clinical Physiologist and could use for intervention allocation.

The revised grading tool using a physiological basis offers a more precise numerical grading, which is both objective and repeatable. This could not only help the Clinical Physiologist to grade there result according to the propose grading scale but probably it also support the surgeon to ascertain the level of severity and could help to decide on either a conservative or surgical approach to treatment. Please note that this research was made to amend the grading for Clinical Physiologist. Although surgeons have to take their own decision for the treatment of CTS, but if they want to consider the treatment on the basis of the proposed nerve conduction study grading, this will probably aid defence of their decisions for the court. This is advisable (but not necessary to follow) that Surgeons could consider proposed Grade 1-2 for physiotherapy treatment, Grade 3-4 for conservative or intervention of steroid treatment and Grade 57 for surgical intervention where the chances of full recovery. Surgeon could decide for surgical intervention of Grade 8 cases, whether it would be beneficial or not in keeping with the patient's age and other medical history.
Future studies looking at prognosis may be helpful in looking at the outcomes from different interventions for those with different gradings of severity and to look at the implications of motor involvement compared with just sensory fascicle involvement. Collections of data are under process for post surgery CTS outcome which will publish later after approval from BCUHB research committee.

\section{Abbreviations \\ ANS: Association of Neurophysiological Scientists; APB: Abductor polices braves; BCUHB: Betsi Cadwaladr University Health Board; BSCN: British Society for Clinical Neurophysiology; CTS: Carpal tunnel syndrome; CV: Conduction velocity; DML: Distal Motor Latency; GPs: General Practices; MCV: Motor conduction velocity; MNAP: Motor nerve action potentials; NCS: Nerve Conduction Studies; NMA: Normal motor amplitude; NSA: Normal sensory amplitude; SCV: Sensory conduction velocity; SNAP: Sensory nerve action potentials}

\section{Acknowledgements}

The author would like to acknowledge and thank Mrs.Julie Evans, Dr. Gareth Payne, and Dr. Bashir Kassam for their encouragement, guidance and help with this study.

\section{Written consent from participants}

A written consent was obtained from all participants and filed in patient notes and a copy kept in the department.

\section{Authors' contributions \\ Salim Hirani carried out the nerve conduction studies, participated in the sequence alignment, drafted the manuscript, design the stufy, performed the statical analysis and carried out the immunoassays. Dr Gareth Payne participated in the sequence alignment. Dr Gareth Payne and Dr Basheer Kassam participated for proof reading. Mrs Julie Evance suported for time allocation in collecting the data analysis. All authors read and approved the final manuscript.}

\section{Funding}

Betsi Cadwaladr University Health Board (BCUHB) funded this study for publication. Awarded Number: 1770146124202136 . The role of the funding body is to support financially in publication this research paper. The Author contributed by the collection, analysis and interpretation of data and in writing the manuscript.

\section{Availability of data and materials}

The datasets analyzed during the current study are not publicly available as they are held within patient records but are available from the

corresponding author on request.

\section{Ethics approval}

We confirm that we have read the Journal's position on issues involved in ethical publication and affirm that this report is consistent with those guidelines. Ethical approval for the research project was obtained from the Heath Research Authority National Research Ethics Service London - Queen Square Research Ethics Committee (Reference 17/LO/0750).

\section{Consent for publication}

Not Applicable.

\section{Competing interests}

The authors declare that they have no competing interests.

Received: 15 March 2019 Accepted: 1 November 2019

Published online: 03 December 2019

\section{References}

1. Ajeena IM, Al-Saad RH, Al-Mudhafar A, Hadi NR, Al-Mridhy SH. Ultrasonic sssessment of females with carpal tunnel syndrome proved by nerve conduction study. Neural Plast. 2013;754564:1-6. 
2. Alfonso C, Jann S, Massa R, Torreggiani A. Diagnosis, treatment and follow-up of th carpal tunnel syndrome: a review. Neurol Sci. 2010;3:243-52.

3. Giannini F, Cioni R, Mondelli M, Padua R, Gregori B, D'Amico P, et al. A new clinical scale of carpal tunnel syndrome: validation of the measurement and clinical-neurophysiological assesment. Clin Neurophysiol. 2002;113(1):71-7.

4. Padua L, Monaco ML, Valente EH, Tonali PA. A useful electrophysiologic parameter for diagnosis of carpal tunnel syndrome. Muscle Nerve. 1996; 19(1):48-53.

5. Campbell EDR. The carpal tunnel syndrome: investigation and assessment of treatment. Proc R Soc Med. 1962;55(5):401-5.

6. Bland J. A neurophysiological grading scale for carpal tunnel syndrome. Muscle Nerve. 2000;1:1280-3.

7. Carvalho FN, Carneiro AP, Paulinelli RR, Carvelho TN. Neurophysiological classification of the carpal tunnel syndrome. Acta Fisiatrica. 2007:4:190-5.

8. Jeong $\mathrm{DH}$, Kim $\mathrm{CH}$. The quantitative relationship between physical examination and the nerve conduction of the carpal tunnel syndrome in patients with and without a diabetic polyneuropathy. Ann Rehabil Med. 2014;38(1):57-63.

9. Jerosh-Herold C, Shepstone L, Wilson ECF, Dyer T, Blake J. Clinical course, costs and predictive factors for response to treatment in carpal tunnel syndrome: the PALMS study protocol. BMC Musculoskelet Disord. 2014; 15(35):1-7.

10. Ozben S, Acar H, Gunaydin S. The second Lumbrical-interosseous latency Comparision in carpal tunnel syndrome. Clin Neurophysiol. 2012;29(3):263-7.

11. AAEM, AAN, AAPMR. Prectice parameter for electrodiagnostic studies in carpal tunnel syndrome: summary statement. Muscle Nerve. 1993b;16:1390-1.

12. Preston D, Logigian EL. Lumbrical and interossei recording in carpal tunnel syndrome. Muscle Nerve. 1992;15:1253-7.

\section{Publisher's Note}

Springer Nature remains neutral with regard to jurisdictional claims in published maps and institutional affiliations.

Ready to submit your research? Choose BMC and benefit from:

- fast, convenient online submission

- thorough peer review by experienced researchers in your field

- rapid publication on acceptance

- support for research data, including large and complex data types

- gold Open Access which fosters wider collaboration and increased citations

- maximum visibility for your research: over $100 \mathrm{M}$ website views per year

At $\mathrm{BMC}$, research is always in progress.

Learn more biomedcentral.com/submissions 